\title{
A case of acute disseminated encephalomyelitis
}

\author{
DSDS Jayaratne ${ }^{1}$, Athula Dissanayake ${ }^{2}$ \\ ${ }^{1}$ Registrar in Medicine, ${ }^{2}$ Consultant Neurologist, Karapitiya Teaching Hospital, Galle.
}

\section{Case report}

A 27 year old female was admitted with acute onset difficulty in walking, diplopia and dysarthria. Symptoms got progressively worse over a period of twenty four hours. She has had a febrile illness two weeks prior to the present illness.

On admission she had nystagmus, cerebellar and pyramidal tract signs and gait ataxia. By next day she became drowsy and developed external ophthalmoplegia. Weakness of lower limbs progressed to grade two.

Her haematological and serological investigations were normal.

CT scan brain revealed enlargement of the brain stem. MRI brain scan revealed hyperintense lesions in brainstem, cerebellum and in cerebral hemispheres subcortically and bilaterally in $\mathrm{T} 2$ weighted images (T2WIs). Thalamic and caudate nuclei lesions were also found.

The clinical picture and the MRI scan findings were suggestive of acute disseminated encephalomyelitis (ADEM). She was started on high dose IV steroids. She made a dramatic improvement over the next few weeks and was able to walk well at the end of four weeks.

\section{Discussion}

ADEM is a monophasic, multifocal inflammatory condition involving the cerebrum, cerebellum, brain stem, optic nerve and spinal cord [1,2,3]. It typically occurs following a viral infection or vaccination over days, weeks or months, punctuated by an acute worsening $[1,2,3]$. Perivenular inflammation (with mainly of $\mathrm{T}$ lymphocytes) and demyelination is the hallmark of the disease [3]. MRI brain scan is the investigation of choice which shows high intensity lesions on T2WIs most commonly in the subcortical white matter, brain stem and cerebellum bilaterally.
However there are no generally accepted diagnostic criteria for ADEM. The predominant white matter involvement suggests demyelination. Whether it was the first episode of Multiple Sclerosis (MS) needs to be considered here, as it has important therapeutic and prognostic implications.

History of recent infection, short duration of illness prior to admission, simultaneous widespread multifocal involvement on MRI brain scan, and the dramatic response to steroids favour the diagnosis of ADEM.

CSF lymphocytic pleocytosis is a feature of ADEM [3]. Her CSF analysis, done during the recovery phase, showed 15 Lymphocytes and a protein content of $40 \mathrm{mg} / \mathrm{dL}$.

Bilateral symmetrical subcortical white matter involvement along with grey matter involvement on MRI brain scan is typical of ADEM, while the grey matter is generally spared in MS [4]. Thalamic involvement is extremely rare in MS. 98\% of MS cases show periventricular white matter involvement which was not seen in this patient [4].

However, distinguishing ADEM from MS on a single MRI brain scan is impossible, and the monophasic course in ADEM has to be demonstrated with follow up of the patient $[4,5]$. Serial MRI scans may prove helpful. Emergence of new lesions and a multiphasic course is highly suggestive of MS, though recurrent ADEM has been reported [1,4,5]. MRI scan done six weeks later in our patient, showed complete resolution of lesions.

The first line therapy is with IV methyl prednisolone. ACTH, IVIG, plasmapheresis and cyclophosphamide have been used as second line treatment. Except for its fulminant form (Hurst Syndrome) prognosis has been favourable in the recent past probably due to steroid treatment $[4,5]$. 
ADEM is rare in adults. This case highlights the need to be aware of this uncommon condition in both children and adults, because of the important therapeutic implications. Early recognition and performance of a MRI brain scan is the key to early diagnosis and prompt treatment.
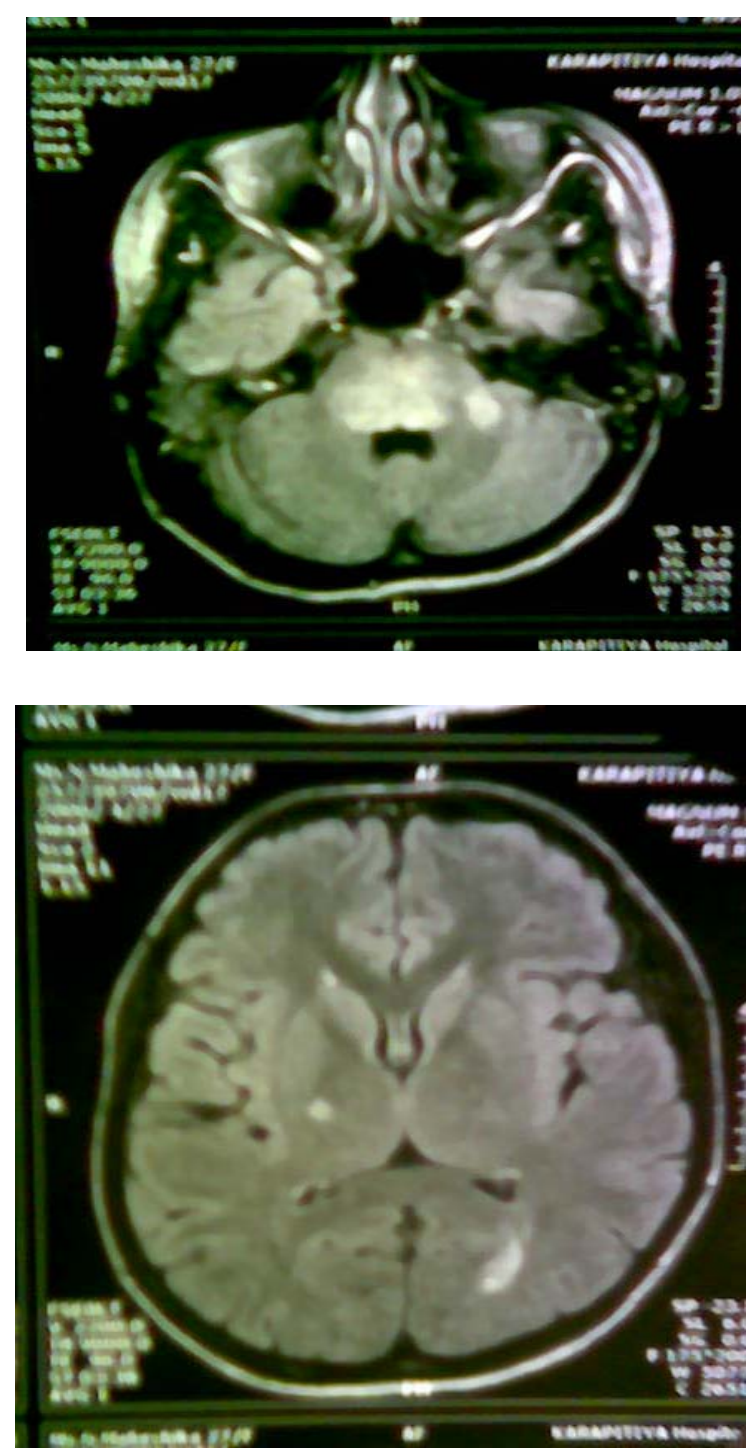

MRI scans of brain of the patient, showing hyper intense lesions in mid brain, thalamus and subcortical white matter.

\section{References}

1. Tailor Rbinson RD. Grand rounds, Hammersmith Hospital. Distinguish ADEM from MS. British Medical Journal 1996; 313: 802-4.

2. Nancy Lee Haris. Case record of Massachusetts GH. New England Journal of Medicine 2002; 347: 1433-40.

3. Stephen L, Hauser, Douglas S, Goodin. MS and other demyelinating diseases. Harrison's Principles of Internal Medicine. $16^{\text {th }}$ edition. Edited by Dennis L. Kasper, 2462-71.

4. Hynson JL. Clinical and neuroradiologic features of acute disseminated encephalomyelitis in children. Neurology 2001; 56: 1308-12.

5. Schwarz S. Acute disseminated encephalomyelitis. A follow up study of 40 adult patients. Neurology 2001; 56: $1313-8$ 